International Journal of Pure and Applied Mathematics

Volume 104 No. 4 2015, 621-630

ISSN: 1311-8080 (printed version); ISSN: 1314-3395 (on-line version)

url: http://www.ijpam.eu

doi: http://dx.doi.org/10.12732/ijpam.v104i4.12

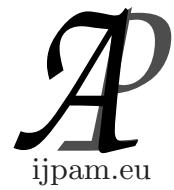

\title{
ON THE SIMPLE EXPANSION OF GENERALIZED TOPOLOGIES
}

\author{
P.M. Dhanya ${ }^{1}$, P.T. Ramachandran ${ }^{2}$ \\ ${ }^{1,2}$ Department of Mathematics \\ University of Calicut \\ Thenjipalam P.O., Malappuram, Kerala, 673635, INDIA
}

\begin{abstract}
In this paper we characterized upper neighbors of a generalized topology in the lattice $L G T(X, L)$, of generalized topologies on a fixed set $X$ and investigated the properties of simple expansion.
\end{abstract}

AMS Subject Classification: 54A05, 06B30

Key Words: generalized topology, simple expansion, upper neighbor, $\mu-$ $T_{o}, \mu-T_{1}, \mu-T_{2}, \mu$-regular, $\mu$-normal, $\mu$-second countable and $\mu$-separable

\section{Preliminaries}

A generalized topology[1] on $X$ is a collection $\mu$ of subsets of $X$ such that $\emptyset \in \mu$ and arbitrary union of elements in $\mu$ is again in $\mu$. If $X \in \mu$, then $\mu$ is said to be a strong generalized topology on $X$. Consider the collection $L G T(X, L)$ of all generalized topologies on a fixed set $X$, it is a complete lattice[6]. A generalized topology $\mu^{\prime}$ is said to be a cover or upper neighbor[5] of $\mu \in L G T(X, L)$ if $\mu \subsetneq \mu^{\prime}$ and if $\mu \subseteq \mu^{\prime \prime} \subseteq \mu^{\prime}$, for some $\mu^{\prime \prime} \in L G T(X, L)$, then $\mu^{\prime \prime}=\mu$ or $\mu^{\prime \prime}=\mu^{\prime}$. Simple expansion of $\mu$ by $A \subseteq X$ is the smallest generalized topology on $X$ containing $\mu$ and $A$. By [5], the simple expansion of $\mu$ by $A, \mu(A)=\mu \cup\{G \cup A: G \in \mu\}$.

Let us go through some basic definitions which are taken from[2].

Received: August 16, 2015

(c) 2015 Academic Publications, Ltd.

${ }^{\S}$ Correspondence author url: www.acadpubl.eu 
Definition 1.1. A generalized topological space $(X, \mu)$ is said to be $\mu-T_{o}$ if for every $x, y \in X$ there exists a set $U \in \mu$ such that either $U \cap\{x, y\}=\{x\}$ or $U \cap\{x, y\}=\{y\}$.

Definition 1.2. A generalized topological space $(X, \mu)$ is said to be $\mu-T_{1}$ if there exist sets $U, V \in \mu$ such that $U \cap\{x, y\}=\{x\}$ and $V \cap\{x, y\}=\{y\}$.

Definition 1.3. A generalized topological space $(X, \mu)$ is said to be $\mu-T_{2}$ if for every $x, y \in X$ there exist disjoint open sets $U, V \in \mu$ such that $x \in U$ and $y \in V$.

Definition 1.4. A generalized topological space $(X, \mu)$ is said to be $\mu$ regular if for every $x \in X$ and a closed set $F$, not containing $x$, there exist disjoint open sets $U, V$ such that $x \in U$ and $F \subseteq V$.

Definition 1.5. A generalized topological space $(X, \mu)$ is said to be $\mu$ normal if for every pair of disjoint closed sets $F, H$, there exist disjoint open sets $U, V$ such that $F \subseteq U$ and $H \subseteq V$.

Definition 1.6. [3] A generalized topological space $(X, \mu)$ is said to be a $\mu$ second countable space if there is a countable base for the generalized topology $\mu$ and $(X, \mu)$ is said to be $\mu$-separable if $X$ contains a countable dense subset.

\section{Notations}

Consider the generalized topological space $(X, \mu)$ on a set $X$. Let $A \subseteq X$. Throughout this paper,

1. $\mu(A)$ denotes the simple expansion of $\mu$ by $A$.

2. $A_{\mu}^{o}$ denotes the interior of $A$ with respect to $\mu$.

3. $\overline{A_{\mu}}$ denotes the closure of $A$ with respect to $\mu$.

4. $A^{c}$ denotes the set theoretic complement of $A$.

5. $\mu \cap A=\{G \cap A: G \in \mu\}$.

6. $|A|$ denotes the cardinality of $A$.

\section{Characterization of Upper Neighbors of $\operatorname{LGT}(X, L)$}

Here we obtain several characterization theorems for a cover of a generalized topology in $\operatorname{LGT}(X, L)$. In paper[5] we saw the result that if $\mu$ is a strong 
generalized topology on a finite set $X$, then for $A \notin \mu$, the simple expansion $\mu(A)$ is a cover of $\mu$ if and only if $\mu(A)=\mu \cup\{A\}$. In this section we prove that this result still holds if $X$ is infinite.

Theorem 2.1. Let $\mu, \mu^{\prime}$ are generalized topologies on $X$. Then $\mu^{\prime}$ is a cover of $\mu$ if and only if $\mu^{\prime}=\mu(A)$ for every $A \in \mu^{\prime} \backslash \mu$.

Proof. Suppose $\mu^{\prime}$ is a cover of $\mu$. Let $A \in \mu^{\prime} \backslash \mu$. Then $\mu(A)$ is the smallest generalized topology containing $\mu$ and $A$ and hence $\mu(A) \subseteq \mu^{\prime}$. Thus $\mu \subseteq$ $\mu(A) \subseteq \mu^{\prime}$. Since $\mu^{\prime}$ is a cover of $\mu, \mu(A)=\mu^{\prime}$. $A$ is arbitrary implying $\mu(A)=\mu^{\prime}$ for every $A \in \mu^{\prime} \backslash \mu$. Now assume $\mu^{\prime}=\mu(A)$ for every $A \in \mu^{\prime} \backslash \mu$. If $\mu^{\prime}$ is not a cover of $\mu$, then there exists a generalized topology $\mu^{\prime \prime}$ on $X$ such that $\mu \subseteq \mu^{\prime \prime} \subseteq \mu^{\prime}$. If $\mu \neq \mu^{\prime \prime}$, then there exists a set $B \in \mu^{\prime \prime}$ such that $B \notin \mu$. Also $B \in \mu^{\prime}$ since $\mu^{\prime \prime} \subset \mu$. But by assumption $\mu^{\prime}=\mu(B)$, hence $\mu^{\prime}$ is the smallest generalized topology containing $\mu$ and $B$. Thus $\mu^{\prime \prime}=\mu^{\prime}$ and hence proving $\mu^{\prime}$ is a cover of $\mu$.

Proposition 2.1. Let $\mu$ be a generalized topology on a set $X$.

1. If $X \notin \mu$, then $\mu(X)=\mu \cup\{X\}$ is always a cover of $\mu$.

2. If $X$ is finite and if $\mu$ is a strong generalized topology on $X$, then for every $A \subset X$ such that $|A|=|X|-1, \mu(A)$ is always a cover of $\mu$.

3. Let $A \subset X$ and $A \notin \mu$. Then if for every $G \in \mu$ suppose either $A \subseteq G$ or $G \subseteq A$, then $\mu(A)$ is a cover of $\mu$.

Proof. This can be easily verified by the reader.

Theorem 2.2. Let $\mu$ be a generalized topology on a set $X$ and $A \notin \mu$. Then for every $G \in \mu$, the simple expansion $\mu(A)$ is finer than $\mu(G \cup A)$.

Proof. Let $G \in \mu$, then $G \cup A \in \mu(A)$. Also $\mu(G \cup A)$ is the smallest generalized topology containing $\mu$ and $G \cup A$, implying $\mu(G \cup A) \subset \mu(A)$. Hence the result.

Theorem 2.3. Let $(X, \mu)$ be a generalized topological space and $A, B$ are subsets of $X$ such that $A, B \notin \mu$. Then,

1. the simple expansion $\mu(B)$ is finer than the simple expansion $\mu(A)$ if and only if $A=G \cup B$ for some $G \in \mu$.

2. the simple expansion $\mu(B)$ is equal to the simple expansion $\mu(A)$ if and only if $A=B$. 
Proof. 1. First assume $\mu(A) \subseteq \mu(B)$, then $A \in \mu(B)$. Since $A \notin \mu$, $A=G \cup B$ for some $G \in \mu$. Conversely if $A=G \cup B$ for some $G \in \mu$, then $A \in \mu(B)$ thus implying $\mu(A) \subseteq \mu(B)$.

2. Use (1).

Corollary 2.1. Let $(X, \mu)$ be a generalized topological space. Then for every $A \subseteq X$ such that $A \notin \mu$, the simple expansion $\mu\left(A \backslash A^{0}\right)$ is always finer than $\mu(A)$.

Proof. The set $A$ can be written as $A=\left(A \backslash A^{0}\right) \cup A^{0}$. Then result follows from Theorem 2.3.

Corollary 2.2. Let $A, B$ are subsets of $X$ such that $A, B \notin \mu$, where $(X, \mu)$ is a generalized topological space. If the simple expansion of $\mu$ by $B$ is finer than the simple expansion of $\mu$ by $A$, then $B$ is a subset of $A$.

Proof. Assume $\mu(A) \subseteq \mu(B)$, then $A \in \mu(B)$. Since $A \notin \mu, A=G \cup B$ for some $G \in \mu$ which implies $B \subseteq A$. Hence the result.

Remark 2.1. Converse of Corollory 2.2 is not true.

For example, let $X=\{a, b, c, d\}$. Consider the generalized topology $\mu=$ $\{\emptyset,\{a, b\},\{a, c\},\{a, b, c\}\}$. Let $A=\{a, d\}$ and $B=\{a\}$. Here $B \subset A$, but $\mu(A)=\mu \cup\{\{a, d\},\{a, b, d\},\{a, c, d\}, X\}$ and $\mu(B)=\mu \cup\{\{a\}\}$. Thus we see that $\mu(B)$ and $\mu(A)$ are not even comparable.

Theorem 2.4. Let $(X, \mu)$ be a generalized topological space. Let $A \subseteq X$ and $A \notin \mu$ and $G \in \mu$. Then the following are equivalent.

1. The simple expansion $\mu(A)$ is a cover of $\mu$.

2. $\mu(A)=\mu \cup\{A\}$.

3. $G \cup\left(A \backslash A^{0}\right) \in \mu(A) \backslash \mu \Rightarrow G=A^{0}$.

4. $G \cap A^{c} \neq \emptyset \Rightarrow G \cup A \in \mu$.

5. $\mu(A)=\mu(B)$ for every $B \in \mu(A) \backslash \mu$.

Proof. $(1) \Leftrightarrow(5)$ by Theorem 2.1 .

$(1) \Rightarrow(2)$ : By Theorem 2.3, for $A, B$ subsets of $X$ and $A, B \notin \mu, \mu(A)=$ $\mu(B)$ if and only if $A=B$. But since $\mu(A)$ is a cover and by $(5), \mu(A) \backslash \mu$ cannot have elements other than $A$. Hence $\mu(A)=\mu \cup\{A\}$. 
$(2) \Rightarrow(1)$ is obvious.

$(2) \Leftrightarrow(3)$ is clear.

$(2) \Rightarrow(4)$ : Assume $\mu(A)=\mu \cup\{A\}$. Now $G \cap A^{c} \neq \emptyset \Rightarrow G \nsubseteq A \Rightarrow G \cup A \neq A$ and $G \cup A \in \mu(A)=\mu \cup\{A\}$ proving $G \cup A \in \mu$.

(4) $\Rightarrow(2)$ : Consider $\mu(A)=\mu \cup\{G \cup A: G \in \mu\}$. If $G \cap A^{c}=\emptyset$ then $G \subseteq A$ and thus $G \cup A=A$. Also if $G \cap A^{c} \neq \emptyset$, then $G \cup A \in \mu$. Thus in either case $G \cup A \in \mu \cup\{A\}$. Hence $\mu(A)=\mu \cup\{A\}$.

Remark 2.2. Every generalized topological space may not have an upper neighbor. For example,

1. Let $X$ be any infinite set and $x \in X$. Define $\mu=\{G: G \subseteq X$ and $x \notin$ $G$ or $x \in G$ and $G^{c}$ is finite $\}$. Then $\mu$ is a generalized topology on $X$. Let $A \subseteq G$ and $A \notin \mu$, then $x \in A$ and $A^{c}$ is infinite. Also $\{y\} \in \mu$ for every $y \in A^{c}$, then $A \cup\{y\} \in \mu(A)$ for every $y \in A^{c}$ resulting $\mu(A) \neq$ $\mu \cup\{A\}$. Since $A \subseteq X$ is arbitrary, $(X, \mu)$ does not have an upper neighbor.

2. Consider the following generalized topology on the set of real numbers $\mathbb{R}$ [5].

$\mu=\{\emptyset, \mathbb{R}\} \cup P(\mathbb{Q}) \cup\{X \cup Y: X=\mathbb{Q} \backslash F, F \subset \mathbb{Q}, F$ is finite, $Y \subset \mathbb{R} \backslash \mathbb{Q}\}$ where $P(\mathbb{Q})$ denotes power set of $\mathbb{Q}$, where $\mathbb{Q}$ denotes the set of all rational numbers.

Let $A \subseteq \mathbb{R}$ and $A \notin \mu$, then $A$ can be

(1) $A=\mathbb{R} \backslash \mathbb{Q}$

then $\mu(A)=\mu \cup\{A \cup X: X \subset \mathbb{Q}\} \neq \mu \cup\{A\}$.

(2) $A=G \cup H$, where $G \subset \mathbb{Q}, G^{c} \cap \mathbb{Q}$ is infinite and $H \subset \mathbb{R} \backslash \mathbb{Q}, H \neq \emptyset$ then $A \cup\{x\} \in \mu(A)$ for every $x \in G^{c} \cap \mathbb{Q}$ implying $\mu(A) \neq \mu \cup\{A\}$.

In either case $\mu(A) \neq \mu \cup\{A\}$. Thus $\mu$ has no upper neighbor.

Norman Levine called a topology $\tau$ on a set $X$ as a superset topology[4] if and only if for $\emptyset \neq O \subseteq A \subseteq X$ and $O \in \tau$, then $A \in \tau$. We generalize this concept and discuss the upper neighbors of superset generalized topology.

Definition 2.1. A generalized topological space $(X, \mu)$ is said to be a superset generalized topological space if, whenever $G \in \mu$ and $G \subset H \subseteq X$, then $H \in \mu$.

Theorem 2.5. Let $(X, \mu)$ be a generalized topological space. Then $\mu$ is a superset generalized topology on $X$ if and only if for every $A \subseteq X$ and $A \notin \mu$, $\mu(A)$ is a cover of $\mu$. 
Proof. Suppose $\mu$ is a superset generalized topology on $X$. Let $A \subseteq X$ and $A \notin \mu, \mu(A)=\{G \cup A: G \in \mu\} \cup \mu$. Since $G \cup A$ is a superset of $G \in \mu$, $G \cup A \in \mu$. Then $G \cup A \in \mu$ for every $G \in \mu$ implying $\mu(A)=\mu \cup\{A\}$ proving $\mu(A)$ is a cover of $\mu$.

Now assume $\mu(A)$ is a cover of $\mu$ for every $A \subseteq X$ and $A \notin \mu$. That is $\mu(A)=\mu \cup\{A\}$ for every $A \subseteq X$ and $A \notin \mu$. Let $G \in \mu$ and $G \subseteq H \subseteq X$. Suppose $H \notin \mu$. Take $A=H \backslash G$, then $A \notin \mu$, otherwise, if $A \in \mu$, then $A \cup G=H \in \mu$, which is a contradiction to our assumption. Now consider the simple expansion of $\mu$ by $A=H \backslash G$. Then $A$ and $H \in \mu(A) \backslash \mu$ implying that $\mu(A) \neq \mu \cup\{A\}$, a contradiction to our assumption that $\mu(A)$ is a cover for every $A \notin \mu$. Hence $H \in \mu$. Thus $\mu$ is a superset generalized topology on $X$.

Remark 2.3. Consider the lattice $L T(X, L)$ of topologies and lattice $L G T(X, L)$ of generalized topologies on a set $X$. Let $\tau$ be a topology on $X$. Then $\tau \in L T(X, L)$ and $\tau \in L G T(X, L)$. Suppose $\tau$ has upper neighbors in $L T(X)$ and $L G T(X, L)$. Then upper neighbor of $\tau$ in $L T(X, L)$ and $L G T(X, L)$ are same if and only if there exists a subset $A \subseteq X$ and $A \notin \tau$ such that for $G \in \tau$, If $G \cap\left(A \backslash A^{0}\right) \neq \emptyset$ then $A \subseteq G$ and If $G \cap A^{c} \neq \emptyset$ then $G \cup A \in \tau$. This is clear from the fact that immediate successor of $\tau$ in $\operatorname{LT}(X, L)$ and $\operatorname{LGT}(X, L)$ are same if and only if there exists $A \subseteq X$ and $A \notin \tau$ such that $\tau(A)=\tau \cup\{A\}$ and use the following theorem.

Theorem 2.6. [4] Let $(X, \tau)$ be a topological space and $A$ is a nonempty subset of $X$ such that $A \notin \tau$. Then a necessary and sufficient condition for the simple expansion topology $\tau(A)$ is the union of the topology $\tau$ and the set $A$ is that

1. $O \in \tau, O \cap\left(A \backslash A^{o}\right) \neq \emptyset \Rightarrow A \subset O$ and

2. $O \cap A^{c} \neq \emptyset \Rightarrow O \cup A \in \tau$.

Remark 2.4. Let $\mu$ be a generalized topological space on a set $X$, which is not a topology. Then there exists an upper neighbor of $\mu$, say $\mu^{\prime}$, which is a topology if and only if the set $\{G \cap H \notin \mu: G, H \in \mu\}$ is a singleton set and moreover $\mu^{\prime}=\mu(G \cap H)=\mu \cup\{G \cap H\}$ where $G, H \in \mu$ such that $G \cap H \notin \mu$.

The following theorem is used to prove our next result.

Theorem 2.7. [2] A generalized topological space $(X, \mu)$ is $\mu-T_{1}$ if and only if for each $x \in M_{\mu},\{x\} \cup\left(X \backslash M_{\mu}\right)$ is a closed set, where $M_{\mu}$ is the union of all open sets in $X$. 
Theorem 2.8. Every non $\mu-T_{1}$ generalized topological space has an upper neighbor.

Proof. Let $(X, \mu)$ be a non $\mu-T_{1}$ generalized topological space. If $X \notin \mu$, then $\mu(X)$ is an upper neighbor of $\mu$. If $X \in \mu$, then since $\mu$ is non $T_{1}$ by Theorem 2.7, there exists an $x \in X$ such that $\{x\}$ is not closed relative to $\mu$ showing $\{x\}^{c} \notin \mu$.

Claim: $\mu\left(\{x\}^{c}\right)$ is an immediate successor of $\mu$.

Let $G \in \mu, G \cap\left(\{x\}^{c}\right)^{c}=G \cap\{x\} \neq \emptyset$, then $x \in G$ and $G \cup\{x\}=G \in \mu$. Then by Theorem $2.4 \mu\left(\{x\}^{c}\right)$ is an upper neighbor of $\mu$.

\section{Properties of Simple Expansion}

Here we discuss the cases when a simple expansion of a generalized topological space $(X, \mu)$ preserves some property $P$ of $(X, \mu)$.

Theorem 3.1. Let $(X, \mu)$ be a generalized topological space which is $\mu-T_{o}\left(\mu-T_{1}\right.$ or $\left.\mu-T_{2}\right)$. Let $A \subseteq X$ and $A \notin \mu$. Then $(X, \mu(A))$ is $\mu(A)-$ $T_{o}\left(\mu(A)-T_{1}\right.$ or $\left.\mu(A)-T_{2}\right)$.

The above theorem can be easily verified.

Lemma 3.1. Let $(X, \mu)$ be a generalized topological space and $A \subseteq X$ and $A \notin \mu$. Let $B \subseteq X$, then

$$
\begin{aligned}
B_{\mu(A)}^{o} & =B_{\mu}^{o} \cup A & & \text { If } A \subseteq B \\
& =B_{\mu}^{o} & & \text { otherwise. }
\end{aligned}
$$

Proof. The proof is easy.

Lemma 3.2. Let $(X, \mu)$ be a generalized topological space and $A \subseteq X$ and $A \notin \mu$. Let $B \subseteq X$, then

$$
\begin{aligned}
\bar{B}_{\mu(A)} & =\bar{B}_{\mu} \cap A^{c} & & \text { If } A \subseteq B^{c} \\
& =\bar{B}_{\mu} & & \text { otherwise. }
\end{aligned}
$$

Proof. We have $\bar{B}_{\mu(A)}=\left[\left(B^{c}\right)_{\mu(A)}^{o}\right]^{c}$. By Lemma 3.1, $\left(B^{c}\right)_{\mu(A)}^{o}=\left(B^{c}\right)_{\mu}^{o} \cup A$ if $A \subseteq B^{c}$ and $\left(B^{c}\right)_{\mu(A)}^{o}=\left(B^{c}\right)_{\mu}^{o}$ otherwise. Thus $\bar{B}_{\mu(A)}=\left[\left(B^{c}\right)_{\mu}^{o} \cup A\right]^{c}=\bar{B}_{\mu} \cap A^{c}$ if $A \subseteq B^{c}$ and $\bar{B}_{\mu(A)}=\bar{B}_{\mu}$ otherwise. 
Theorem 3.2. Let $(X, \mu)$ be a $\mu$-regular generalized topological space and let $A$ be a subset of $X$ such that $A \notin \mu$ and $A^{c} \in \mu$. Then $(X, \mu(A))$ is $\mu(A)$-regular.

Proof. Let $x \in X$ and $x \notin F$ where $F$ is a closed set in $(X, \mu)$. If $F=O^{c}$ for some $O \in \mu$, then since $(X, \mu)$ is $\mu$-regular, there exists an open set $U \in$ $\mu \subset \mu(A)$ such that $x \in U \subset \bar{U} \subseteq F$. Now if $F=(O \cup A)^{c}$ for some $O \in \mu$, then $x \notin F$ and hence $x \notin(O \cup A)^{c}=O^{c} \cap A^{c}$. Thus $x$ does not belong to $O^{c}$ or $A^{c}$.

Case 1: $\left(x \notin O^{c}\right.$ and $\left.x \notin A^{c}\right)$ or $\left(x \in O^{c}\right.$ and $\left.x \notin A^{c}\right)$

Here $x \in A$ and $F=O^{c} \cap A^{c} \subseteq A^{c}, A$ and $A^{c}$ are open in $(X, \mu(A))$ and $A \cap A^{c}=\emptyset$. Thus $x$ and $F$ can be separated by open sets in $(X, \mu(A))$.

Case 2: $x \notin O^{c}$ and $x \in A^{c}$

$F=O^{c} \cap A^{c} \subseteq O^{c}$ and since $(X, \mu)$ is $\mu$-regular there exist disjoint open sets $U, V$ in $(X, \mu)$ such that $x \in U$ and $O^{c} \subseteq V$. Thus $x \in U$ and $F=O^{c} \cap A^{c} \subseteq V$. Hence $(X, \mu(A))$ is $\mu(A)$-regular.

Lemma 3.3. Let $(X, \mu)$ be a generalized topological space and $A$ be a subset of $X$ such that $A \notin \mu$. Then the generalized topological space $(A, \mu \cap$ $A)=(A, \mu(A) \cap A)$ and $\left(A^{c}, \mu \cap A^{c}\right)=\left(A^{c}, \mu(A) \cap A^{c}\right)$.

Lemma 3.4. Let $(X, \mu)$ be a normal generalized topological space and $F \subseteq X$ be a closed set. Then $(F, \mu \cap F)$ is $\mu \cap F$-normal.

Theorem 3.3. Let $(X, \mu)$ be a $\mu$-normal generalized topological space. Let $A \subseteq X$ be such that $A \notin \mu, A^{c} \in \mu$ and $A \cap G \in \mu(A)$ for every $G \in \mu$. Then $(X, \mu(A))$ is $\mu(A)$-normal if and only if $\left(A^{c}, \mu \cap A^{c}\right)$ is $\mu \cap A^{c}$-normal.

Proof. Assume $(X, \mu(A))$ is $\mu(A)$-normal. $A^{c}$ is closed in $(X, \mu(A))$. We have by Lemma 3.4 closed subspace of a normal space is normal. Thus $\left(A^{c}, \mu(A) \cap\right.$ $\left.A^{c}\right)$ is normal. By Lemma 3.3, $\left(A^{c}, \mu(A) \cap A^{c}\right)=\left(A^{c}, \mu \cap A^{c}\right)$. Hence $\left(A^{c}, \mu \cap A^{c}\right)$ is $\mu \cap A^{c}$-normal.

Now assume the converse. Let $F, G$ are closed and disjoint in $(X, \mu(A))$. Then $F \cap A$ and $G \cap A$ are closed and disjoint in $(X, \mu(A) \cap A)=(A, \mu \cap A)$. Since $A$ is closed in $(X, \mu), F \cap A$ and $G \cap A$ are closed in $(X, \mu)$, which is $\mu$-normal. Thus there exist disjoint open sets $U$ and $V$ such that $F \cap A \subseteq U$ and $G \cap A \subseteq V$. Also $F \cap A^{c}$ and $G \cap A^{c}$ are disjoint and closed in $\left(A^{c}, \mu(A) \cap A^{c}\right)=\left(A^{c}, \mu \cap A^{c}\right)$, which is $\mu \cap A^{c}$-normal. Then there exist disjoint open sets $U^{\prime}$ and $V^{\prime}$ such that $F \cap A^{c} \subseteq U^{\prime}$ and $G \cap A^{c} \subseteq V^{\prime}$. Now $F=(F \cap A) \cup\left(F \cap A^{c}\right) \subseteq(U \cap A) \cup U^{\prime}$ which is open since $A \cap U$ and $U^{\prime}$ are open and hence the union. Similarly $G=(G \cap A) \cup\left(G \cap A^{c}\right) \subseteq(V \cap A) \cup V^{\prime}$ which is also open by the same reason. 
Also $(U \cap A) \cup U^{\prime}$ and $(V \cap A) \cup V^{\prime}$ are disjoint since $(U \cap A)$ and $(V \cap A)$ are disjoint subsets of $A$ and $U^{\prime}$ and $V^{\prime}$ are disjoint subsets of $A^{c}$. Hence $(X, \mu(A))$ is $\mu(A)$-normal.

Theorem 3.4. Let $(X, \mu)$ be a generalized topological space and $A \subseteq X$ and $A \notin \mu$. Then

1. $(X, \mu)$ is $\mu$-second countable if and only if $(X, \mu(A))$ is $\mu(A)$-second countable.

2. $(X, \mu)$ is $\mu$-separable if and only if $(X, \mu(A))$ is $\mu(A)$-separable.

Proof. (1) Assume $(X, \mu(A))$ is $\mu(A)$-second countable. Since $\mu \subset \mu(A)$, $(X, \mu)$ is also $\mu$-second countable. Now let $\left\{G_{n}\right\}_{n \in N}$, where $N$ is the set of all Natural numbers, is a countable collection of open sets in $(X, \mu)$ which forms a basis for $(X, \mu)$. Then $\left\{G_{n}\right\}_{n \in N} \cup\{A\}$ forms a basis for the generalized topological space $(X, \mu(A))$.

(2) Assume the generalized topological space $(X, \mu(A))$ is $\mu(A)$-separable. Then $(X, \mu)$ is $\mu$-separable since $\mu \subseteq \mu(A)$. Now if $(X, \mu)$ is $\mu$-separable, then $(X, \mu)$ has a countable dense subset say $H$, implying $H \cup\{x\}$, where $x \in A$, is a countable dense subset of $(X, \mu(A))$ proving $(X, \mu(A))$ is $\mu(A)$-separable.

Theorem 3.5. Let $(X, \mu)$ be a connected generalized topological space and if $A \notin \mu$, is a dense subset of $(X, \mu)$, then $(X, \mu(A))$ is a connected generalized topological space.

Proof. If $(X, \mu(A))$ is not connected, let $U, V \in \mu(A)$ constitute a separation for $(X, \mu(A))$. Then $U$ and $V$ both cannot be open in $(X, \mu)$ since $(X, \mu)$ is connected and also both cannot belong to the set $\{O \cup A: O \in \mu\}$ for some $O \in \mu$, since $U \cap V=\emptyset$. Therefore let $U \in \mu$ and $V=O \cup A$ where $O \in \mu$. $U \cap V=U \cap(O \cup A)=(U \cap A) \cup(U \cap A) \neq \emptyset$ because $U \cap A \neq \emptyset$ since $A$ is dense in $(X, \mu)$, a contradiction. Hence the result.

Theorem 3.6. Let $(X, \mu)$ be a generalized topological space and $A$ be a connected dense subset of $X$ and $A \notin \mu$. Then $(X, \mu(A))$ is connected.

Proof. Suppose $(X, \mu(A))$ is not connected. Let $U, V \in \mu(A)$ be such that $U \cap V=\emptyset$ and $U \cup V=X$. Then $U \cap A$ and $V \cap A$ are open and disjoint in $(A, \mu(A) \cap A)=(A, \mu \cap A)$ by Lemma 3.3. Clearly $(U \cap A) \cup(V \cap A) \subseteq A$. Now let $x \in A$, then either $x \in U$ or $x \in V$. Assume $x \in U$, then $x \in U \cap A$ and hence $x \in(U \cap A) \cup(V \cap A)$. Thus $A \subseteq(U \cap A) \cup(V \cap A)$. Hence 
$A=(U \cap A) \cup(V \cap A)$, a contradiction, since $A$ is connected. Similarly we get a contradiction if $x \in V$. Hence $(X, \mu(A))$ is connected.

\section{Acknowledgments}

First author expresses sincere thanks to second author Dr. Ramachandran P. T., Associate Professor, Dept. of Mathematics, University of Calicut, for his constant support and critical suggestions through out the preparation of this paper. Financial support from CSIR, Govt. of India is greatly acknowledged by first author.

\section{References}

[1] Á. Császár, Generalized Topology, Generalized Continuity, Acta Math. Hungar., 96 (2002), 351-357, doi: 10.1023/A:1019713018007.

[2] GE Xun, GE Ying, $\mu$-Separations in Generalized Topological Spaces Appl. Math. J. Chinese Univ. 25 No.2,(2010), 243-252, doi:10.1007/s11766-010$2274-1$.

[3] J. B. T. Ayawan , S. R. Canoy, Jr., Axioms of Countability in Generalized Topological Spaces, International Mathematical Forum, 8, No. 31(2013), 1523 - 1530, doi: http://dx.doi.org/10.12988/imf.2013.37142.

[4] Levine, Norman, Minimal Simple Extensions of Topologies, Kyungpook Math J., 19, No. 1(1979), 43-55.

[5] P. M. Dhanya, On the Lattice of Generalized Topologies, Int. J. Pure. Appl. Math. 102, No. 1, (2015) 85-95, doi: http://dx.doi.org/10.12732/ijpam.v102i1.8.

[6] R. Baskaran, M. Murugalingam, and D. Sivaraj, Lattice of Generalized Topologies, Acta Math. Hungar., 133, No.4, (2011) 365-371, doi: 10.1007/s10474-011-0085-3. 